\title{
Investigation of Bufavirus and Parvovirus 4 in Patients with Gastro-Enteritis from the South-East of France
}

\author{
Francis Simo-Fouda ${ }^{1}$, Laurence Thirion ${ }^{1}{ }^{(D)}$, Antoine Nougairède ${ }^{1}$, Léa Luciani ${ }^{1}$, Jean-Sélim Driouich ${ }^{1}$, \\ Paul Rémi Petit ${ }^{1}$, Pascal Delaunay ${ }^{2}$ and Remi N. Charrel ${ }^{1, *(1)}$
}

1 Unité des Virus Émergents (UVE), Aix-Marseille Université, IRD 190-Inserm 1207, 13005 Marseille, France; francis-girauld.simo-fouda@etu.univ-amu.fr (F.S.-F.); laurence.thirion@ird.fr (L.T.); antoine.nougairede@univ-amu.fr (A.N.); lea.luciani@ap-hm.fr (L.L.); jean-selim.driouich@etu.univ-amu.fr (J.-S.D.); paul-remi.peti@@etu.univ-amu.fr (P.R.P.)

2 Inserm U1065 Centre Méditerranéen de Médecine Moléculaire, Département de Parasitologie-Mycologie, Centre Hospitalier Universitaire de Nice (CHU Nice), 06202 Nice, France; delaunay.p@chu-nice.fr

* Correspondence: remi.charrel@univ-amu.fr

check for updates

Citation: Simo-Fouda, F.; Thirion, L.; Nougairède, A.; Luciani, L.; Driouich, J.-S.; Petit, P.R.; Delaunay, P.; Charrel, R.N. Investigation of Bufavirus and Parvovirus 4 in Patients with Gastro-Enteritis from the South-East of France. Pathogens 2021, 10, 1151. https://doi.org/10.3390/ pathogens10091151

Academic Editor: Magda Dunowska

Received: 26 July 2021

Accepted: 4 September 2021

Published: 7 September 2021

Publisher's Note: MDPI stays neutral with regard to jurisdictional claims in published maps and institutional affiliations.

Copyright: (c) 2021 by the authors. Licensee MDPI, Basel, Switzerland. This article is an open access article distributed under the terms and conditions of the Creative Commons Attribution (CC BY) license (https:/ / creativecommons.org/licenses/by/ $4.0 /)$.

\begin{abstract}
Bufavirus (BuV) and human parvovirus 4 (PARV4) belong to the Parvoviridae family. We assessed BuV and PARV4 DNA presence by real-time PCR analysis in stool, blood and respiratory samples collected in patients from Marseille and Nice, two large cities in the South-East of France. BuV DNA was detected in diarrheic stool samples from 92 patients (3.6\% of 2583 patients), particularly men and adults, and patients from the nephrology and the infectious disease departments. Among the patients with a BuV-positive stool sample and for whom at least one blood sample was available ( $n=30$ patients), BuV DNA was detected also in 3 blood samples. In contrast, BuV DNA was not detected in any of the respiratory samples from 23 patients with BuV-positive stool. BuV detection rate was comparable in stool samples from patients with and without diarrhea. We did not detect PARV4 DNA in any of the stool specimens ( $n=2583$ patients). Our results suggest that PARV4 fecal-oral transmission is rare or non-existent in the South-East of France while BuV circulates with a relatively high rate in this area.
\end{abstract}

Keywords: bufavirus; human parvovirus 4; Protoparvovirus; parvovirus; Parvoviridae; gastroenteritis; diarrhea; France

\section{Introduction}

Viruses of the Parvoviridae family are small single-stranded DNA viruses that infect a wide range of organisms, from invertebrates to humans. The first human parvovirus was discovered in 1965 and since 2005, many new parvoviruses have been identified in human samples [1,2].

Bufavirus $(\mathrm{BuV})$, a virus from the genus Protoparvovirus, was identified in 2012 from fecal samples of children with diarrhea in Burkina Faso [3,4]. To date, three genotypes, sharing $65-73 \%$ amino acid identity within VP2, have been described [1,5]. BuV DNA has been detected in stool samples (prevalence ranging from 0 to $4 \%$ ), mostly from patients presenting with diarrhea, in many countries. Other populations have been rarely studied and a limited number of patients has been tested: $\mathrm{BuV}$ was not found in blood or cerebrospinal fluids $(n=126)$ in Turkey; Buv DNA was detected in 1/995 in nasal swabs in Finland and 0/44 nasopharyngeal aspirate from Latvia; studies conducted in Finland, Turkey and Thailand detected BuV DNA in diarrheic samples but not in non-diarrheic samples, whereas studies in Bangladesh and Malawi reported BuV DNA in both diarrheic and non-diarrheic samples [6-12]. The causative role of $\mathrm{BuV}$ in gastroenteritis remains unclear, and its association with other clinical presentations remains largely to be explored $[5,6,8]$.

Human parvovirus 4 (PARV4), a virus belonging to the genus Tetraparvovirus was first described in 2005 in plasma from patients with acute viral infection syndrome. It has been 
classified in three genotypes, and genotype 2 was formerly known as PARV5 $[5,13,14]$. The low genetic diversity between PARV4 genotypes $(<3 \%$ at the amino acid level) supports that introduction in human populations is likely to have occurred recently [15]. PARV4 has been detected in blood/plasma samples, autopsy samples, stools, nasopharyngeal swabs, bone marrow and cerebrospinal fluid; the clinical impact of PARV4 infection remains uncertain, but reported disease associations include influenza-like syndrome, encephalitis, worsening of HIV evolution and fetal hydrops; PARV4 association with gastroenteritis remains to be established $[5,13,15,16]$.

The aims of the present study were (i) to investigate BuV and PARV4 presence in stool samples from patients with diarrhea in the South-East of France; (ii) to check whether in patients with positive stools, both viruses can be found also in blood and respiratory samples; (iii) to determine whether the detection rate of these viruses varies in patients with and without diarrhea in hospital settings.

\section{Results and Discussion}

\subsection{BuV and PARV4 Detection in Stool Samples from Patients with Diarrhea (Cohort 1)}

\subsubsection{Parvovirus 4 Detection}

None of the 3148 stool samples of cohort 1 contained PARV4 DNA. Although PARV4 has been detected in stool samples in Ghana ( $0.53 \%, 5 / 943$ fecal samples from children) [17], our results suggest that PARV4 fecal-oral transmission is rare or non-existent in the SouthEast of France.

\subsubsection{Bufavirus Detection}

Demographic Data

The median age of the 2583 patients of cohort 1 was 56 years (range 0 to 101 years) and $1305(50.5 \%)$ were men (Table 1$)$.

Table 1. Socio-demographic characteristics of the patients in cohort 1.

\begin{tabular}{|c|c|c|c|}
\hline & $\begin{array}{c}\text { BuV DNA + Patients } \\
(N=92)\end{array}$ & $p$-Value & $\begin{array}{l}\text { Study Population } \\
\qquad(N=2583)\end{array}$ \\
\hline \multicolumn{4}{|l|}{ Residence, $n(\%)$} \\
\hline Marseille & $83(3.5)$ & & 2375 \\
\hline Nice & $9(4.3)$ & & 208 \\
\hline \multicolumn{4}{|l|}{ Sex, $n(\%)$} \\
\hline Men & $69(5.3)$ & & 1305 \\
\hline Women & $23(1.8)$ & & 1278 \\
\hline Sex Ratio (M/W) & 3.00 & $<0.0001$ & 1.02 \\
\hline \multicolumn{4}{|l|}{ Age, Years } \\
\hline Median & 58 & & 56 \\
\hline Range & $1-92$ & & $0-101$ \\
\hline Children ( $\leq 15$ years $)$ & $4(0.7)$ & & 539 \\
\hline \multirow[t]{2}{*}{ Adults ( $>15$ years) } & $88(4.3)$ & & 2044 \\
\hline & & $<0.0001$ & \\
\hline Age/Sex Groups, $n(\%)$ & & & \\
\hline Young ( $\leq 15$-Year-old) Men & $4(1.4)$ & & 286 \\
\hline Young ( $\leq 15$-Year-old) Women & $0(0.0)$ & & 253 \\
\hline Adult (>15-Year-old) Men & $65(6.4)$ & & 1019 \\
\hline \multirow[t]{2}{*}{ Adult (>15-Year-old) Women } & $23(2.2)$ & & 1025 \\
\hline & & $<0.0001$ & \\
\hline \multicolumn{4}{|l|}{ Seasonality; $n$ (\%) } \\
\hline Cumulated Winters & $36(3.17)$ & & 1136 \\
\hline Cumulated Springs & $26(3.45)$ & & 754 \\
\hline Cumulated Summers & $14(3.33)$ & & 420 \\
\hline \multirow[t]{2}{*}{ Cumulated Autumns } & $31(3.70)$ & & 838 \\
\hline & & 0.94 & \\
\hline
\end{tabular}


Samples (stool, blood and respiratory specimens) were tested for the presence of $\mathrm{BuV}$ DNA using a real-time quantitative PCR assay described by one of the leading research group in the field [18]. In total, BuV DNA was detected in $3.6 \%$ of patients $(n=92 / 2583$, $n=107$ stool samples) of cohort 1 (Table 1 ). This positivity rate is similar to the one reported by Smits et al. in the Netherlands (3.7\%) [19], but it is higher than observed in Finland (1.1\%) [18], China (1.7\%) [20] and Thailand (0.3\%) [10]. The difference in BuV detection rate could be affected by method used for samples testing, as we used realtime PCR with positivity cutoff at a cycle threshold of $<40$, while in Netherland authors used viral metagenomics approach, or nested PCR in Thailand; in Finland and China authors used real-time PCR without detailing the cut-off value [10,18-20]. Moreover, a study by the French Reference Center for gastroenteritis viruses reported a detection rate of $15.8 \%$ ( $n=85 / 538$ stool samples) in children admitted to the Dijon and Saint-Etienne University Hospital Centers in 2019 [21]. If confirmed, this and our results suggest that $\mathrm{BuV}$ is circulating at higher rate in France than in other countries.

The age of BuV-positive patients ranged from 1 to 92 years (median: 58 years). BuV detection rate in $>15$-year-old patients $(4.3 \%, 88 / 2044)$ was significantly higher than in $\leq 15$-year-old patients $(0.7 \%, 4 / 539, p<0.0001)$ (Table 1$)$. Similar epidemiological trends were reported in Finland, in the Netherlands, in China and in Thailand. For instance, in Thailand, the BuV DNA detection rate in children was $0.07 \%$ (one case) compared with $3.7 \%$ in adults [10].

The men/women ratio was 3 in the $\mathrm{BuV}$-positive group, and $\mathrm{BuV}$ detection rate was significantly higher in men than women $(5.3 \%, 69 / 1305$, vs. $1.8 \%, 23 / 1278 ; p<0.0001)$, as observed in Finland (sex ratio of 2.5) [18]. In other studies, the sex differences in detection rate were not significant $[10,19,20]$.

Our results did not suggest that $\mathrm{BuV}$ is a seasonal virus (Table 1), in agreement with previous data [18]. In contrast, temporal clustering associated with the cold season was observed in China and in Turkey [11,20].

\section{Characteristics of BuV-Positive Patients}

The $\mathrm{BuV}$ detection rate was higher in patients from the Nephrology $(9.3 \%, 19 / 204$ stool samples from 18 patients) and Infectious Disease (6.0\%, 13/215 stool samples from 12 patients) departments compared with other departments (detection rates $<5 \%$ ).

The viral load was determined in the $107 \mathrm{BuV}$-positive stool samples collected from 92 patients and ranged from $3.8 \times 10^{3}$ to $4.2 \times 10^{8}$ genome copies per gram of stool (or per $\mathrm{mL}$ of $15 \%$ fecal suspension), with mean and median values of $7.1 \times 10^{6}$ and $2.8 \times 10^{4}$ genome copies per gram of stool (Figure 1). Previous studies reported values that were lower in stool samples $[9,18,19]$, with values ranging between $2.1 \times 10^{2}-1.6 \times 10^{3}$ [9] and $1.9 \times 10^{3}-3.2 \times 10^{4}[18]$ copies $/ \mathrm{mL}$ of $10 \%$ fecal suspension. In addition, $23.4 \%(25 / 107)$ of our samples had a viral load $\geq 1.1 \times 10^{5}$ genome copies per gram of stool. Previous studies showed other viruses causing gastroenteritis displayed similar viral loads. For instance, a report on children with gastroenteritis found that the adenovirus median viral load was $4.56 \times 10^{3}$ genome copies per gram of stools (range $2.14 \times 10^{2}-1.32 \times 10^{5}$ ) [22]. Another study on patients with gastroenteritis showed that norovirus G1 median viral load was $8.4 \times 10^{5}$ genome copies per gram of stools (range: $2.2 \times 10^{4}-2.9 \times 10^{10}$ ) [23]. These data suggest that $\mathrm{BuV}$ might be a diarrhea-causing virus in some patients who should be identified in specific studies.

$\mathrm{BuV}$ could lead to chronic infection or carriage. Among the 11 patients with available sequential stool samples, five (\#1,\#14, \#19, \#38 and \#82) (Table 2) had BuV-positive samples for a period $\geq 3$ weeks $(26,27,49,62$ and 245 days, respectively). 


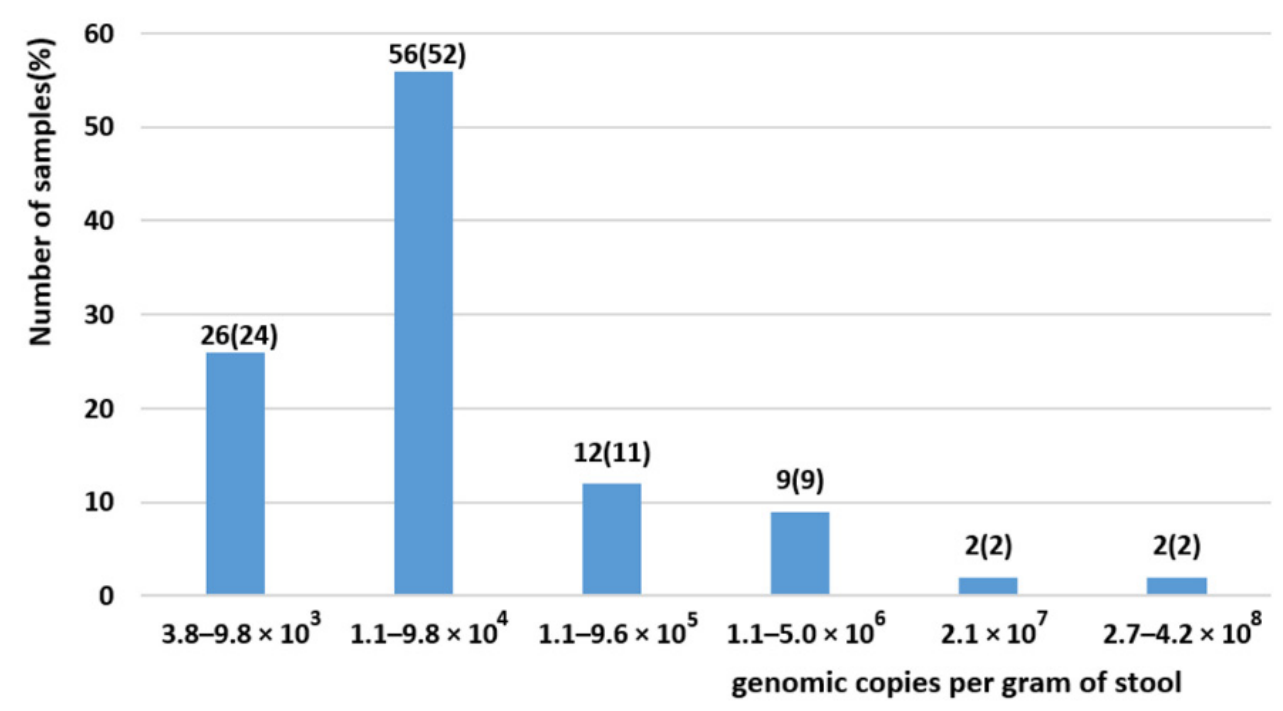

Figure 1. BuV viral load in the $107 \mathrm{BuV}$-positive stool samples.

Table 2. BuV viral load in stool samples and blood samples (cohort 1).

\begin{tabular}{|c|c|c|c|c|c|}
\hline N. & Sex & $\begin{array}{c}\text { N of } \\
\text { Days * }\end{array}$ & $\begin{array}{l}\text { BuV Viral Load in } \\
\text { Stool Samples }\end{array}$ & $\begin{array}{l}\text { Others Viruses } \\
\text { Found in Stool }\end{array}$ & $\begin{array}{l}\text { Blood Samples *** } \\
\text { (Days before Death) }\end{array}$ \\
\hline \multirow{6}{*}{1} & \multirow{6}{*}{$M$} & 1 & - & - & Negative (64) \\
\hline & & 9 & $4.2 \times 10^{8}$ & $\begin{array}{c}A D V^{\dagger} \text { and Noro }{ }_{\text {GII }}^{\ddagger} \\
\text { a }\end{array}$ & - \\
\hline & & 57 & - & - & $2.0 \times 10^{4}(8)$ \\
\hline & & 58 & $2.7 \times 10^{8}$ & $A D V$ and Noro GII & - \\
\hline & & 61 & - & - & $3.3 \times 10^{3}(4)$ \\
\hline & & 64 & - & - & $5.8 \times 10^{3}(1)$ \\
\hline 2 & M & - & $2.1 \times 10^{7}$ & - & - \\
\hline 3 & $\mathrm{M}$ & - & $2.1 \times 10^{7}$ & - & - \\
\hline 4 & M & 1 & $5.0 \times 10^{6}$ & ADV & Negative \\
\hline 5 & $\mathrm{~F}$ & - & $4.6 \times 10^{6}$ & - & - \\
\hline 6 & $\mathrm{~F}$ & - & $2.7 \times 10^{6}$ & - & - \\
\hline 7 & M & - & $2.4 \times 10^{6}$ & - & - \\
\hline 8 & $\mathrm{M}$ & - & $1.7 \times 10^{6}$ & - & - \\
\hline \multirow{3}{*}{9} & \multirow{3}{*}{$\mathrm{M}$} & 1 & $1.7 \times 10^{6}$ & - & - \\
\hline & & 10 & $6.8 \times 10^{5}$ & - & - \\
\hline & & 14 & - & - & Negative \\
\hline 10 & $\mathrm{M}$ & - & $1.6 \times 10^{6}$ & - & - \\
\hline 11 & $\mathrm{~F}$ & - & $1.1 \times 10^{6}$ & - & - \\
\hline 12 & $\mathrm{M}$ & - & $9.6 \times 10^{5}$ & - & - \\
\hline 13 & $\mathrm{M}$ & 1 & $9.1 \times 10^{5}$ & - & Negative \\
\hline \multirow{4}{*}{14} & \multirow{4}{*}{$M$} & 1 & - & - & Negative (82) \\
\hline & & 5 & $8.6 \times 10^{5}$ & - & - \\
\hline & & 22 & - & - & Negative (61) \\
\hline & & 32 & $2.8 \times 10^{4}$ & - & - \\
\hline \multirow{2}{*}{15} & \multirow{2}{*}{$\mathrm{M}$} & 1 & $7.9 \times 10^{5}$ & - & - \\
\hline & & 8 & - & - & Negative \\
\hline
\end{tabular}


Table 2. Cont.

\begin{tabular}{|c|c|c|c|c|c|}
\hline N. & Sex & $\begin{array}{c}\text { Nof } \\
\text { Days * }\end{array}$ & $\begin{array}{l}\text { BuV Viral Load in } \\
\text { Stool Samples ** }\end{array}$ & $\begin{array}{l}\text { Others Viruses } \\
\text { Found in Stool }\end{array}$ & $\begin{array}{l}\text { Blood Samples } * * * \\
\text { (Days before Death) }\end{array}$ \\
\hline \multirow[b]{2}{*}{16} & \multirow{2}{*}{$\mathrm{M}$} & 1 & - & - & Negative \\
\hline & & 3 & $4.6 \times 10^{5}$ & - & - \\
\hline 17 & M & - & $2.7 \times 10^{5}$ & - & - \\
\hline 18 & $\mathrm{~F}$ & - & $2.3 \times 10^{5}$ & - & - \\
\hline \multirow{4}{*}{19} & \multirow{4}{*}{$\mathbf{F}$} & 1 & $1.7 \times 10^{5}$ & - & - \\
\hline & & 189 & $4.7 \times 10^{4}$ & - & - \\
\hline & & 189 & $3.0 \times 10^{4}$ & - & - \\
\hline & & 245 & $1.1 \times 10^{6}$ & - & - \\
\hline \multirow{2}{*}{20} & \multirow{2}{*}{$\mathrm{M}$} & 1 & $1.6 \times 10^{5}$ & - & - \\
\hline & & 1 & $3.8 \times 10^{4}$ & - & - \\
\hline 21 & $M$ & - & $1.3 \times 10^{5}$ & - & - \\
\hline 22 & $\mathrm{~F}$ & - & $9.8 \times 10^{4}$ & ADV & - \\
\hline 23 & $\mathrm{M}$ & - & $8.6 \times 10^{4}$ & - & - \\
\hline \multirow{2}{*}{24} & \multirow{2}{*}{$\mathrm{M}$} & 1 & $8.4 \times 10^{4}$ & - & - \\
\hline & & 6 & $3.0 \times 10^{4}$ & - & - \\
\hline 25 & $\mathrm{~F}$ & - & $8.3 \times 10^{4}$ & - & - \\
\hline 26 & $\mathrm{M}$ & - & $6.3 \times 10^{4}$ & - & - \\
\hline 27 & $\mathrm{M}$ & - & $5.6 \times 10^{4}$ & ADV & - \\
\hline 28 & $\mathrm{M}$ & - & $5.4 \times 10^{4}$ & - & - \\
\hline 29 & $M$ & - & $5.3 \times 10^{4}$ & - & - \\
\hline 30 & $\mathrm{~F}$ & - & $5.3 \times 10^{4}$ & - & - \\
\hline 31 & M & - & $5.2 \times 10^{4}$ & - & - \\
\hline \multirow{2}{*}{32} & \multirow{2}{*}{$M$} & 1 & $5.0 \times 10^{4}$ & - & - \\
\hline & & 215 & - & - & Negative (16) \\
\hline 33 & $\mathrm{~F}$ & - & $4.9 \times 10^{4}$ & - & - \\
\hline 34 & $\mathrm{M}$ & - & $4.7 \times 10^{4}$ & - & - \\
\hline \multirow{2}{*}{35} & \multirow{2}{*}{$\mathrm{M}$} & 1 & - & - & Negative \\
\hline & & 5 & $4.6 \times 10^{4}$ & - & - \\
\hline 36 & $\mathrm{M}$ & - & $4.5 \times 10^{4}$ & - & - \\
\hline \multirow{3}{*}{37} & \multirow{3}{*}{$\mathrm{M}$} & 1 & $4.1 \times 10^{4}$ & - & - \\
\hline & & 2 & - & - & Negative \\
\hline & & 67 & Negative & - & \\
\hline \multirow{2}{*}{38} & \multirow{2}{*}{$\mathbf{M}$} & 1 & $3.9 \times 10^{4}$ & - & - \\
\hline & & 26 & $7.1 \times 10^{3}$ & - & - \\
\hline 39 & $\mathrm{M}$ & 1 & $3.8 \times 10^{4}$ & - & Negative \\
\hline \multirow{2}{*}{40} & \multirow{2}{*}{$\mathrm{F}$} & 1 & - & - & Negative \\
\hline & & 2 & $3.3 \times 10^{4}$ & - & - \\
\hline 41 & $F$ & - & $3.2 \times 10^{4}$ & - & - \\
\hline 42 & M & 1 & $3.1 \times 10^{4}$ & - & Negative \\
\hline 43 & $\mathrm{~F}$ & - & $3.0 \times 10^{4}$ & - & - \\
\hline \multirow{3}{*}{44} & \multirow{3}{*}{$\mathrm{M}$} & 1 & - & - & $1.2 \times 10^{3}$ \\
\hline & & 4 & $3.0 \times 10^{4}$ & - & - \\
\hline & & 9 & $2.3 \times 10^{4}$ & - & \\
\hline
\end{tabular}


Table 2. Cont.

\begin{tabular}{|c|c|c|c|c|c|}
\hline N. & Sex & $\begin{array}{c}\text { N of } \\
\text { Days * }\end{array}$ & $\begin{array}{l}\text { BuV Viral Load in } \\
\text { Stool Samples ** }\end{array}$ & $\begin{array}{l}\text { Others Viruses } \\
\text { Found in Stool }\end{array}$ & $\begin{array}{l}\text { Blood Samples } * * * \\
\text { (Days before Death) }\end{array}$ \\
\hline 45 & $\mathrm{~F}$ & - & $2.9 \times 10^{4}$ & - & - \\
\hline 46 & $M$ & - & $2.8 \times 10^{4}$ & - & - \\
\hline 47 & $\mathrm{M}$ & - & $2.7 \times 10^{4}$ & - & - \\
\hline 48 & $\mathrm{~F}$ & - & $2.6 \times 10^{4}$ & - & - \\
\hline 49 & $M$ & - & $2.3 \times 10^{4}$ & - & - \\
\hline 50 & $\mathrm{M}$ & - & $2.2 \times 10^{4}$ & ADV & - \\
\hline 51 & M & $\begin{array}{l}1 \\
7\end{array}$ & $\begin{array}{c}2.2 \times 10^{4} \\
-\end{array}$ & $\begin{array}{c}\text { ADV } \\
-\end{array}$ & $\begin{array}{c}- \\
\text { Negative }\end{array}$ \\
\hline 52 & $\mathrm{~F}$ & $\begin{array}{l}1 \\
5 \\
5\end{array}$ & $\begin{array}{c}- \\
2.0 \times 10^{4} \\
1.7 \times 10^{4}\end{array}$ & $\begin{array}{l}- \\
- \\
-\end{array}$ & $\begin{array}{c}\text { Negative } \\
- \\
-\end{array}$ \\
\hline 53 & $\mathrm{M}$ & - & $1.9 \times 10^{4}$ & - & - \\
\hline 54 & M & $\begin{array}{l}1 \\
7 \\
\end{array}$ & $\begin{array}{l}1.7 \times 10^{4} \\
1.8 \times 10^{4}\end{array}$ & $\begin{array}{l}- \\
-\end{array}$ & $\begin{array}{l}- \\
-\end{array}$ \\
\hline 55 & M & $\begin{array}{l}1 \\
4\end{array}$ & $1.7 \times 10^{4}$ & - & $\begin{array}{c}\text { Negative } \\
-\end{array}$ \\
\hline 56 & $M$ & - & $1.5 \times 10^{4}$ & - & - \\
\hline 57 & M & - & $1.5 \times 10^{4}$ & - & - \\
\hline 58 & $\mathrm{~F}$ & $\begin{array}{c}1 \\
417 \\
\end{array}$ & $\begin{array}{l}1.4 \times 10^{4} \\
\text { Negative }\end{array}$ & - & - \\
\hline 59 & $\mathrm{M}$ & 1 & $1.4 \times 10^{4}$ & - & Negative \\
\hline 60 & $\mathrm{~F}$ & - & $1.4 \times 10^{4}$ & - & - \\
\hline 61 & $\mathrm{~F}$ & - & $1.3 \times 10^{4}$ & - & - \\
\hline 62 & $\mathrm{M}$ & $\begin{array}{l}1 \\
2\end{array}$ & $\begin{array}{c}- \\
1.3 \times 10^{4}\end{array}$ & - & $\begin{array}{c}\text { Negative } \\
-\end{array}$ \\
\hline 63 & $\mathrm{M}$ & - & $1.3 \times 10^{4}$ & - & - \\
\hline 64 & $\mathrm{~F}$ & $\begin{array}{l}1 \\
6\end{array}$ & $\begin{array}{l}1.2 \times 10^{4} \\
1.9 \times 10^{4}\end{array}$ & - & - \\
\hline 65 & $\mathrm{M}$ & $\begin{array}{c}1 \\
16\end{array}$ & $\begin{array}{c}- \\
1.2 \times 10^{4}\end{array}$ & - & $\begin{array}{c}\text { Negative } \\
-\end{array}$ \\
\hline 66 & $\mathrm{M}$ & - & $1.2 \times 10^{4}$ & - & - \\
\hline 67 & $\mathrm{~F}$ & - & $1.1 \times 10^{4}$ & - & - \\
\hline 68 & $\mathrm{M}$ & - & $9.8 \times 10^{3}$ & - & - \\
\hline 69 & $\mathrm{M}$ & 1 & $9.8 \times 10^{3}$ & Norovirus GII & Negative \\
\hline 70 & $\mathrm{~F}$ & - & $9.3 \times 10^{3}$ & - & - \\
\hline 71 & $\mathrm{M}$ & - & $9.2 \times 10^{3}$ & - & - \\
\hline 72 & $\mathrm{M}$ & $\begin{array}{l}1 \\
3 \\
\end{array}$ & $\begin{array}{c}8.7 \times 10^{3} \\
-\end{array}$ & - & $\begin{array}{c}- \\
\text { Negative }\end{array}$ \\
\hline 73 & $\mathrm{M}$ & 1 & $8.2 \times 10^{3}$ & Norovirus GI & Negative \\
\hline 74 & $M$ & - & $8.1 \times 10^{3}$ & - & - \\
\hline 75 & M & $\begin{array}{c}1 \\
12 \\
\end{array}$ & $\begin{array}{c}- \\
8.1 \times 10^{3}\end{array}$ & - & $\begin{array}{c}\text { Negative } \\
-\end{array}$ \\
\hline 76 & $\mathrm{M}$ & - & $7.9 \times 10^{3}$ & Astrovirus & - \\
\hline
\end{tabular}


Table 2. Cont.

\begin{tabular}{|c|c|c|c|c|c|}
\hline N. & Sex & $\begin{array}{c}\text { N of } \\
\text { Days * }\end{array}$ & $\begin{array}{l}\text { BuV Viral Load in } \\
\text { Stool Samples ** }\end{array}$ & $\begin{array}{l}\text { Others Viruses } \\
\text { Found in Stool }\end{array}$ & $\begin{array}{l}\text { Blood Samples } * * * \\
\text { (Days before Death) }\end{array}$ \\
\hline \multirow{4}{*}{77} & \multirow{4}{*}{$M$} & 1 & - & - & Negative (41) \\
\hline & & 5 & $7.7 \times 10^{3}$ & - & Negative (37) \\
\hline & & 9 & Negative & - & - \\
\hline & & 38 & - & - & Negative (4) \\
\hline \multirow[t]{3}{*}{78} & \multirow[t]{3}{*}{$\mathrm{M}$} & 1 & - & - & Negative \\
\hline & & 2 & Negative & - & - \\
\hline & & 3 & $7.6 \times 10^{3}$ & - & - \\
\hline \multirow[t]{2}{*}{79} & \multirow[t]{2}{*}{$\mathrm{M}$} & 1 & $7.1 \times 10^{3}$ & - & - \\
\hline & & 14 & $1.1 \times 10^{4}$ & - & - \\
\hline 80 & $\mathrm{M}$ & 1 & $6.5 \times 10^{3}$ & - & Negative \\
\hline 81 & $\mathrm{M}$ & - & $6.5 \times 10^{3}$ & - & - \\
\hline \multirow[t]{4}{*}{82} & \multirow[t]{4}{*}{$M$} & 1 & Negative & - & \\
\hline & & 215 & $6.5 \times 10^{3}$ & - & Negative (83) \\
\hline & & 277 & $1.1 \times 10^{5}$ & - & - \\
\hline & & 284 & - & - & $2.8 \times 10^{3}(15)$ \\
\hline 83 & $\mathrm{~F}$ & - & $6.4 \times 10^{3}$ & - & - \\
\hline 84 & $\mathrm{~F}$ & - & $6.2 \times 10^{3}$ & - & - \\
\hline \multirow{3}{*}{85} & \multirow[t]{3}{*}{$M$} & 1 & - & - & Negative (76) \\
\hline & & 3 & - & - & Negative (74) \\
\hline & & 77 & $6.1 \times 10^{3}$ & - & - \\
\hline \multirow{2}{*}{86} & \multirow{2}{*}{$\mathrm{M}$} & 1 & $5.6 \times 10^{3}$ & - & - \\
\hline & & 4 & Negative & - & \\
\hline 87 & $\mathrm{M}$ & - & $5.6 \times 10^{3}$ & - & - \\
\hline 88 & $\mathrm{M}$ & - & $5.4 \times 10^{3}$ & - & - \\
\hline \multirow{2}{*}{89} & \multirow{2}{*}{$\mathrm{M}$} & 1 & - & - & Negative \\
\hline & & 3 & $5.1 \times 10^{3}$ & - & - \\
\hline 90 & $\mathrm{M}$ & - & $4.7 \times 10^{3}$ & - & - \\
\hline 91 & $M$ & - & $4.1 \times 10^{3}$ & - & - \\
\hline 92 & M & - & $3.8 \times 10^{3}$ & - & - \\
\hline
\end{tabular}

$\mathrm{M}=$ male; $\mathrm{F}=$ female$^{*}{ }^{*}=$ number of days after collection of the first sample (day 1 ; not indicated when only one sample was collected); ${ }^{* *}=$ genome copies per gram of stool; ${ }^{* * *}=$ genome copies per milliliter of blood; ${ }^{\dagger} \mathrm{ADV}=$ adenovirus; ${ }^{\ddagger}$ Noro = norovirus; in italic = deceased patients; in bold = sequential stool samples positives for a period $\geq 3$ weeks.

Three months after the stool samples were collected, the death rate was significantly higher among BuV-positive than BuV-negative patients (14.5\%, 12/83, vs. 7.9\%; 182/2292; $p=0.034)$. One year later, the death rate was still higher among BuV-positive than BuVnegative patients $(16.9 \%, 14 / 83$, vs. $10.8 \%, 247 / 2292)$, but this difference was not significant $(p=0.10)$. The causes of death of these $14 \mathrm{BuV}$-positive patients were cancer (\#21, \#29, \#32, $\# 41, \# 74, \# 77$ and \#82), pneumopathy (1\#, \#46, 49\#, 56\# and 85\#), mediastinitis (\#14) and vascular infection complication (\#91). Comorbidities, particularly kidney failure (7/14), were recorded for 11 of these 14 patients. The mortality rates according to the age class are shown in Figure 2. 


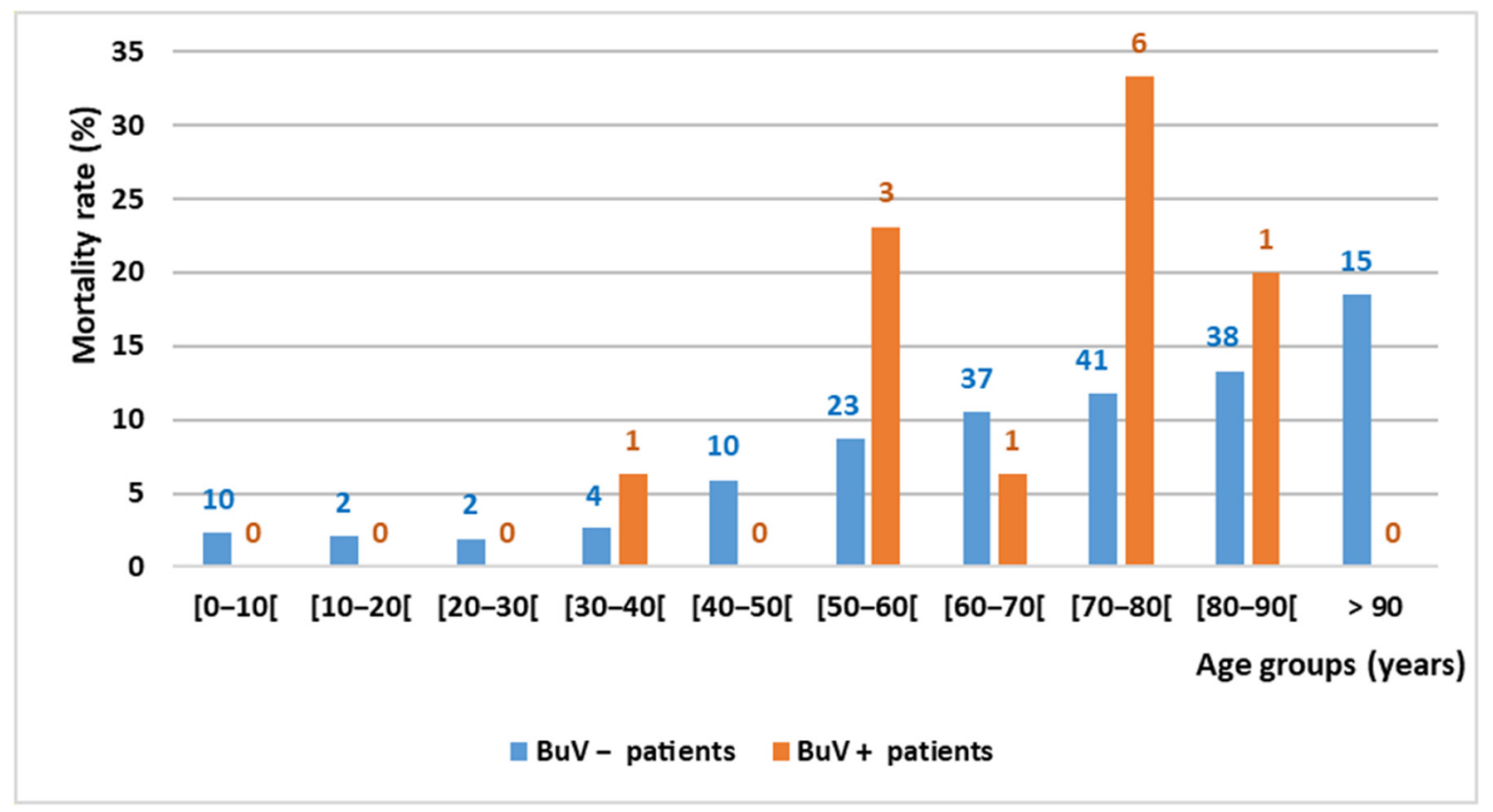

Figure 2. Mortality rate by age group and BuV DNA status in Marseille (3 months after stool sample collection). The number above each column indicates the number of deceased patients.

$\mathrm{BuV}$ has been discovered recently, and data on whether it can cause other diseases or syndrome are limited [1]. Indeed, this is the first time that data from $>10 \mathrm{BuV}$-positive patients are used to investigate $\mathrm{BuV}$ infection. We do not know why the death rate was higher in BuV-positive than in BuV-negative patients (14.5\% vs. $7.9 \%)$, and if confirmed, should be thoroughly investigated. Data on fatal outcome among BuV-positive patients are scarce. Chieochansin et al. reported that among four BuV-positive patients in Thailand one 90-year-old patient died due to congestive heart failure [10]. This underlines the need of additional studies. As 7/14 of deceased patients in our study had cancer, the link between $\mathrm{BuV}$ and cancer also should be explored. However, the possibility that $\mathrm{BuV}$ acquisition or prolonged shedding may be facilitated by the failing conditions encountered in immunocompromised patients should also be considered. Interestingly, cutavirus, a close $\mathrm{BuV}$ relative, has been detected in skin biopsies of patients with cutaneous lymphoma $(4 / 17$, France) and malignant skin lesions (1/10, Denmark). Moreover, such as $\mathrm{BuV}$, it has been found in stool samples from patients with diarrhea (4/245 Brazil; 1/100 Botswana) [24-26].

\section{BuV Co-Infection}

Among the $107 \mathrm{BuV}$-positive stool samples, adenovirus was co-detected in 7 samples (6 patients), norovirus GII in 3 samples (2 patients), norovirus GI and astrovirus in 1 sample/each. BuV, adenovirus and norovirus GII were simultaneously detected in the stool sample of a HIV-positive patient. Conversely, rotavirus and sapovirus were not detected in the BuV-positive stool samples. Several previous studies reported coinfection of $\mathrm{BuV}$ with norovirus, although co-infection with astrovirus was the first to be reported $[9,11,18,20,27,28]$. Co-infection of bufavirus and adenovirus could be explored once we know that members of the Dependoparvovirus genus in the same family compensate for their genetic limitations by co-opting helper viruses, most commonly adenoviruses or herpesviruses [5].

\subsection{BuV Testing in Blood and Respiratory Samples from Patients with Buv-Positive Stool Samples}

Among the 14 patients with BuV-positive stool samples who died, blood samples were available for 6 of them (14 samples). BuV DNA was detected in blood samples of two of these patients, with a viral load ranging from $2.8 \times 10^{3}$ to $2.0 \times 10^{4}$ genome copies $/ \mathrm{mL}$ of blood (Table 2). In BuV-positive patients with available blood samples, $\mathrm{BuV}$ was more 
frequently detected in blood samples of patients who died (2/6 patients vs. 1/24 patients) (Table 2). In Switzerland, BuV nucleic acids were detected in $1 / 25$ plasma samples; this patient presented with acute leukemia who underwent bone marrow transplantation and the issue was fatal 2 months later; the same patient had also a BuV-positive stool sample [29]. Together with our results, this supports that $\mathrm{BuV}$ could be involved in extra-intestinal clinical manifestations. Last, specific antibodies were detected in $13.2 \%$ of patients in another region of France [30], and in $1.9 \%, 3.6 \%, 56.1 \%, 72.3 \%$ and $84.8 \%$ of patients from cohorts from Finland, USA, Iran, Kenya and Iraq, respectively [2].

Finally, 27 oral and respiratory samples ( 8 saliva, 6 pleural fluids, 6 bronchoalveolar lavages, 4 nasopharyngeal aspirations and 3 sputum) were available for 23 of the $83 \mathrm{BuV}$-positive patients from Marseille. All these samples were BuV-negative by PCR, in agreement with results in Finland where only one of 955 nasal swab specimens was positive [9]. Whether $\mathrm{BuV}$ can cause respiratory infection can clearly be questioned and justifies further studies designed specifically to lift the veil.

\subsection{BuV Detection Rate in Stool Samples from Patients with and without Diarrhea (Cohort 2)}

In cohort 2 (30-75-years-old patients), BuV DNA positivity was not significantly different in patients with and without diarrhea: $10.50 \%$ vs. $10.00 \%$ ( $p$-value $=1)$ for men, and $4.59 \%$ vs. $4.41 \%(p$-value $=1)$ for women (Table 3). Among the BuV-positive samples, only one from the group with diarrhea, was also positive for another pathogen (Tropheryma whipplei).

Table 3. BuV DNA detection rate in stool samples of adult patients with and without diarrhea, collected from November 2020 to April 2021 (Marseille).

\begin{tabular}{clcc}
\hline & & BuV + Patients & $\begin{array}{c}\text { Study Population } \\
\text { (30-75 Years of Age) }\end{array}$ \\
\hline Patients with & Men & $23(10.50)$ & 219 \\
Diarrhea; $n(\%)$ & Women & $5(4.59)$ & 109 \\
$N=328$ & Men/Women Ratio & $23 / 5(4.60)$ & $219 / 109(2.01)$ \\
& Age, Range (Median), Years & $30-75(60)$ & $30-75(61)$ \\
\hline & Men & $11(10.00)$ & 110 \\
Patients without & Women & $6(4.41)$ & 136 \\
Diarrhea; $n(\%)$ & Men/Women Ratio & $11 / 6(1.83)$ & $110 / 136(0.81)$ \\
$N=246$ & Age, Range (Median), Years & $33-74(61)$ & $30-75(53)$ \\
\hline
\end{tabular}

Data on BuV DNA detection in adult populations without diarrhea are scarce. In Thailand, 15 adults without diarrhea were all BuV-negative, whereas $3 / 81$ adult patients with diarrhea were $\mathrm{BuV}$-positive [10]. BuV DNA was detected in $1 / 227$ children without diarrhea in Bangladesh [12], and in a cohort of 164 children from Malawi where the unique $\mathrm{BuV}$ DNA-positive sample belonged to the group without diarrhea [6].

Viral loads were higher in stool samples from the group with diarrhea than without diarrhea in cohort 2 (Tables S1 and S2 of supplemental data).

$\mathrm{BuV}$ detection rates in 30-75-year-old men and women of cohort 2 were higher than those obtained for cohort 1 with respectively detection rates of $10.5 \%$ vs. $7.4 \%$ ( $p$-value $=0.2$ ) in men and $4.6 \%$ vs. $3.0 \%$ ( $p$-value $=0.5$ ) in women (Table 4$)$. This could reflect that $\mathrm{BuV}$ circulation might be increasing in Marseille from 2017 to 2021 although the lack of comparability between the two cohort might have introduced a bias. This merit to be confirmed in future studies. 
Table 4. BuV detection rate in 30-75-year-old patients with diarrhea from cohort 1 and cohort 2 (Marseille).

\begin{tabular}{|c|c|c|c|}
\hline & & BuV + Patients & $\begin{array}{c}\text { Study Population } \\
\text { (30-75 Years of Age) }\end{array}$ \\
\hline Cohort 1, & Men & $23(7.4)$ & 311 \\
\hline November & Women & $8(3.0)$ & 267 \\
\hline 2017-April 2018; $n$ (\%) & Men/Women ratio & $23 / 8(2.9)$ & $311 / 267$ (1.2) \\
\hline$N=578$ & Age, range (median), years & $30-75(59)$ & $30-75(60)$ \\
\hline Cohort 2, & Men & $23(10.5)$ & 219 \\
\hline November & Women & $5(4.6)$ & 109 \\
\hline 2020-April 2021; $n(\%)$ & Men/Women ratio & $23 / 5(4.6)$ & $219 / 109(2.0)$ \\
\hline$N=328$ & Age, range (median), years & $30-75(60)$ & $30-75(61)$ \\
\hline
\end{tabular}

\subsection{Genotyping of BuV-Positive Samples}

Genotyping has been attempted for the 92 patients, which had positive results for $\mathrm{BuV}$ detection (Table 2). A total of 54 were identified as BuV-1, 3 as BuV-2 and 11 as BuV-3: so, a total of $68 / 92(74 \%)$ of patients were infected with a single genotype. Another 14 patients showed co-infection with two or three genotypes detected in the same clinical sample; 10 patients were negative for the three genotypes (despite $\mathrm{BuV}$-positive result) and remain to be investigated further.

In conclusion, our study shows that (i) PARV4 fecal-oral transmission is rare or nonexistent in the South-East of France; (ii) $\mathrm{BuV}$ circulates with a relatively high rate in the South-East of France; (iii) BuV circulation is not associated with seasonality; (iv) adults are more frequently infected than children, and men are more frequently infected than women; (v) BuV might lead to chronic infection or carriage in patients without diarrhea; (vi) genotype 1 is largely dominant in south-eastern France.

\section{Materials and Methods}

\subsection{Study Population}

Two cohorts were obtained from Marseille and/or Nice.

Cohort 1 included (i) 2375 patients $(n=2874$ diarrheic stool samples collected between March 2017 and February 2019) from different departments of Marseille University Hospital Center (Emergency, Pediatrics, Internal Medicine, Infectious Diseases, Nephrology, Gastroenterology, Surgery, Hematology and Immunology, Oncology, Cardiovascular Medicine and Geriatrics); (ii) 208 patients ( $n=274$ diarrheic stool samples collected between January 2017 and March 2018) from the Parasitology Department of Nice University Hospital Center. From patients with BuV-positive stool samples in Marseille, blood and respiratory samples were searched for availability in the Biobank; when available. Selection criteria were as follows: blood and respiratory samples were searched approximately 1 month before or after the BuV-positive stool sample was collected.

Cohort 2 included 30-75-year-old patients from different clinical departments of Marseille University Hospital Center. This cohort consisted of 2 groups: the patients with diarrhea (328 patients including 219 men and 109 women, 395 stool samples) and patients without diarrhea (246 patients including 110 men and 136 women, 261 stool samples). Stool samples were collected between November 2020 and April 2021. The hospital records of the 246 patients without diarrhea indicated that 260/261 stool samples were tested mostly for T. whipplei (9/153 positive samples by PCR), and that 5/86 stool samples were positive for other bacteria by culture or PCR (1 Clostridium difficile), 1/30 was positive for Blastocystis hominis and $0 / 8$ were positive for viruses. A similar search for the group with diarrhea allowed obtaining data for 393/395 stool samples: 5/128 were positive for T. whipplei, 27/297 were positive for other bacteria by culture or PCR (14 C. difficile), 2/37 were respectively positive for Giardia intestinalis + Blastocystis hominis and Oestrus ovis and 2/54 were, respectively, positive for Norovirus GI and Cytomegalovirus. 


\subsection{Sample Preparation}

For stool collection, a pea size stool sample (around $33 \mathrm{mg}$ ) or $300 \mu \mathrm{L}$ of liquid stools was taken using an inoculation loop or micropipette. It was then put in a sterile tube containing $1 \mathrm{ml}$ of distilled water and mixed by vortexing before storage at $-20{ }^{\circ} \mathrm{C}$ until use.

After thawing and mixing, approximately $10 \mu \mathrm{L}$ of each stool sample was transferred with an inoculation loop to a conical Falcon tube containing $2 \mathrm{~mL}$ of distilled water and mixed by vortexing. Then $200 \mu \mathrm{L}$ of this suspension was transferred into a S-Block (Qiagen ${ }^{\circledR}$, Venlo, The Netherlands) for nucleic acid extraction with Qiacube (Qiagen ${ }^{\circledR}$ ).

Blood and respiratory samples were collected by clinicians and kept at $-80^{\circ} \mathrm{C}$ until use. Frozen blood samples were thawed and mixed, and then $100 \mu \mathrm{L}$ of blood sample was diluted in $100 \mu \mathrm{L}$ of $0.9 \% \mathrm{NaCl}$, and $200 \mu \mathrm{L}$ of this dilution was transferred into a S-Block $\left(\right.$ Qiagen ${ }^{\circledR}$ ) for nucleic acid extraction with Qiacube $\left(\right.$ Qiagen $\left.{ }^{\circledR}\right)$. Frozen respiratory samples were thawed and mixed, and then $200 \mu \mathrm{L}$ of each sample was transferred into a S-Block $\left(\right.$ Qiagen ${ }^{\circledR}$;) for nucleic acid extraction with Qiacube $\left(\right.$ Qiagen $\left.{ }^{\circledR}\right)$.

\subsection{Nucleic acid Extraction}

DNA was isolated from stool, blood and respiratory samples with the Cador ${ }^{\circledR}$ Pathogen 96 Qiacube HT Kit $\left(\right.$ Qiagen $\left.{ }^{\circledR}\right)$ and the Qiacube HT device (Qiagen ${ }^{\circledR}$ ) following the manufacturer's recommendations with some modifications. For a 96-well plate, VXL lysis buffer included $17 \mathrm{~mL}$ of VXL buffer, $2.2 \mathrm{~mL}$ of proteinase $\mathrm{K}, 100 \mu \mathrm{L}$ of RNA carrier $(1 \mu \mathrm{g} / \mu \mathrm{L})$ and $1 \mathrm{~mL}$ of bacteriophages MS2/T4 as internal control. The AVE Buffer elution volume was $150 \mu \mathrm{L}$ per well for stool samples, and $90 \mu \mathrm{L}$ for blood and respiratory samples.

\subsection{Sample Testing}

By using primers and hydrolysis probes targeting the NS1 region of the virus, BuV presence was tested in all stool samples (cohort 1 and 2) as previously described [18]. The presence of PARV4, genotypes 1 and 2, and PARV4 genotype 3 was tested only in stool samples from cohort 1 by using previously described primers and hydrolysis probes specific to ORF2 region for PARV4 genotypes 1 and 2 [31], and NS1 region for PARV4 genotype 3 [32]. BuV-positive stool samples from cohort 1 were also tested to determine the presence of norovirus GI [33], norovirus GII [34], rotavirus (with the Rota 2 Fwd ACCATCTWCACRTRACCCTCTATGAG, Rota 2 Rev GGTCACATAACGCCCCTATAGC and Rota 2 P FAM-AGTTAAAAGCTAACACTGTCAA-BHQ primers adapted from Freeman M et al., 2008) [35], sapovirus [36], astrovirus [36] and adenovirus [37]. BuV presence was also tested in the available blood and respiratory samples of patients with BuV-positive stool samples (cohort 1). Data from the second cohort have been dedicated to section on BuV detection rate in stool samples from patients with and without diarrhea; all others results sections are based on data of the first cohort.

\subsection{Positive Control}

In-house designed plasmids were used as positive controls for BuV, PARV4 genotypes 1 and 2 , and PARV4 genotype 3 . These plasmids included the BuV/PARV4 DNA sequences targeted by the primers/probes used for pathogen detection, the promoter T7, and BamHI, $\mathrm{XmaI}$ and NotI restriction sites.

\subsection{Taqman Real-Time PCR Assays}

Real-time PCR was performed on a Bio-Rad CFX96 ${ }^{\mathrm{TM}}$ Real-Time System, software version 3.1 (Bio-Rad Laboratories, Hercules, CA, USA), using LightCycler ${ }^{\circledR} 480$ Probes Master (Roche Diagnostics, Germany) and a total volume of $30 \mu \mathrm{L}(10 \mu \mathrm{L}$ of DNA and $20 \mu \mathrm{L}$ of reaction mix). Each reaction mix contained $2.6 \mu \mathrm{L}$ of PCR-grade water, $15 \mu \mathrm{L}$ of LightCycler 480 Probes Master $2 \times, 1 \mu \mathrm{L}(333.3 \mathrm{nM})$ of forward and reverse primers, and $0.4 \mu \mathrm{L}(133.3 \mathrm{nM})$ of probe. The thermal cycle program was: $10 \mathrm{~min}$ at $95^{\circ} \mathrm{C}$, followed by 
45 cycles of $10 \mathrm{sec}$ at $95^{\circ} \mathrm{C}, 30 \mathrm{~s}$ at $60^{\circ} \mathrm{C}$ and $1 \mathrm{~s}$ at $72{ }^{\circ} \mathrm{C}$. Samples were classified as positive at a cycle threshold of $<40$.

Genotyping was performed using 3 specific real-time quantitative PCR assays each targeting one genotype (detailed in Supplementary Table S3).

For quantitation, serial 10 -fold dilutions of the plasmid $\left(2.88 \times 10^{11}\right.$ copies $\left./ \mu \mathrm{L}\right)$ containing the BuV NS1 insert targeted by primers and probes were used to calibrate the Bio-Rad CFX96 ${ }^{\mathrm{TM}}$ Real-Time System. The resulting linear equation and the mean weight of stool samples were used to estimate the viral load with Microsoft Excel 2016.

\subsection{Statistical Analysis}

For data analysis, the Fisher's exact test was used to compare percentages with the SPSS statistical package release 17.0 (SPSS Inc., Chicago, IL, USA).

Supplementary Materials: The following are available online at https:/ /www.mdpi.com/article/ 10.3390/pathogens10091151/s1. Table S1. BuV viral load in diarrheic stools samples (cohort 2). Table S2. BuV viral load in non-diarrheic stools samples (cohort 2). Table S3. Real-time PCR assay used for genotyping BuV-DNA positive samples.

Author Contributions: Conceptualization, R.N.C., P.D., L.T. and F.S.-F.; methodology, L.T., A.N. and F.S.-F.; software, L.T., A.N. and J.-S.D.; validation, R.N.C. and L.T.; formal analysis, F.S.-F.; resources, R.N.C.; writing—original draft preparation, F.S.-F.; writing—-review and editing, R.N.C., L.T., L.L. and P.R.P.; supervision, R.N.C., L.T. and P.D. All authors have read and agreed to the published version of the manuscript.

Funding: F.S.-F. received a scholarship from the foundation Méditerranée Infection.

Institutional Review Board Statement: Not applicable.

Informed Consent Statement: This research work was conducted retrospectively.

Data Availability Statement: All data are provided in the article.

Acknowledgments: This publication was supported by the European Virus Archive GLOBAL (EVAGLOBAL) project that has received funding from the European Union's Horizon 2020 research and innovation program under grant agreement No. 871029. We would like to thank Céline Gazin, Pierre Combe for their excellent technical assistance.

Conflicts of Interest: The authors declare no conflict of interest.

\section{References}

1. Soderlund-Venermo, M. Emerging Human Parvoviruses: The Rocky Road to Fame. Annu. Rev. Virol. 2019, 6, 71-91. [CrossRef] [PubMed]

2. Vaisanen, E.; Mohanraj, U.; Kinnunen, P.M.; Jokelainen, P.; Al-Hello, H.; Barakat, A.M.; Sadeghi, M.; Jalilian, F.A.; Majlesi, A.; Masika, M.; et al. Global Distribution of Human Protoparvoviruses. Emerg. Infect. Dis. 2018, 24, 1292-1299. [CrossRef]

3. Vaisanen, E.; Fu, Y.; Hedman, K.; Soderlund-Venermo, M. Human Protoparvoviruses. Viruses 2017, 9, 354. [CrossRef]

4. Phan, T.G.; Vo, N.P.; Bonkoungou, I.J.; Kapoor, A.; Barro, N.; O’Ryan, M.; Kapusinszky, B.; Wang, C.; Delwart, E. Acute diarrhea in West African children: Diverse enteric viruses and a novel parvovirus genus. J. Virol. 2012, 86, 11024-11030. [CrossRef] [PubMed]

5. Cotmore, S.F.; Agbandje-McKenna, M.; Canuti, M.; Chiorini, J.A.; Eis-Hubinger, A.; Hughes, J.; Mietzsch, M.; Modha, S.; Ogliastro, M.; Pénzes, J.J.; et al. ICTV Virus Taxonomy Profile: Parvoviridae. J. Gen. Virol. 2019, 100, 367-368. [CrossRef] [PubMed]

6. Mohanraj, U.; Jokinen, M.; Thapa, R.R.; Paloniemi, M.; Vesikari, T.; Lappalainen, M.; Tarkka, E.; Nora-Krukle, Z.; Vilmane, A.; Vettenranta, K.; et al. Human Protoparvovirus DNA and IgG in Children and Adults with and without Respiratory or Gastrointestinal Infections. Viruses 2021, 13, 483. [CrossRef]

7. Dapra, V.; Galliano, I.; Montanari, P.; Zaniol, E.; Calvi, C.; Alliaudi, C.; Bergallo, M. Bufavirus, Cosavirus, and Salivirus in Diarrheal Italian Infants. Intervirology 2021, 1-4. [CrossRef]

8. Altay Kocak, A.; Ocal, M.; Polat, M.; Kanik Yuksek, S.; Aktas Tapisiz, A.; Tezer, H.; Ozkul, A.; Ergunay, K.; Bozdayi, G.; Ahmed, K. Multicenter investigation of bufavirus in the etiology of viral central nervous system infections of adults and children. Mikrobiyol Bul. 2017, 51, 191-194. [CrossRef]

9. Vaisanen, E.; Paloniemi, M.; Kuisma, I.; Lithovius, V.; Kumar, A.; Franssila, R.; Ahmed, K.; Delwart, E.; Vesikari, T.; Hedman, K.; et al. Epidemiology of two human protoparvoviruses, bufavirus and tusavirus. Sci. Rep. 2016, 6, 39267. [CrossRef]

10. Chieochansin, T.; Vutithanachot, V.; Theamboonlers, A.; Poovorawan, Y. Bufavirus in fecal specimens of patients with and without diarrhea in Thailand. Arch. Virol. 2015, 160, 1781-1784. [CrossRef] 
11. Altay, A.; Yahiro, T.; Bozdayi, G.; Matsumoto, T.; Sahin, F.; Ozkan, S.; Nishizono, A.; Soderlund-Venermo, M.; Ahmed, K. Bufavirus genotype 3 in Turkish children with severe diarrhoea. Clin. Microbiol. Infect. 2015, 21, 965e1-965e4. [CrossRef] [PubMed]

12. Okitsu, S.; Khamrin, P.; Takanashi, S.; Thongprachum, A.; Hoque, S.A.; Takeuchi, H.; Khan, M.A.; Hasan, S.M.T.; Iwata, T.; Shimizu, H.; et al. Molecular detection of enteric viruses in the stool samples of children without diarrhea in Bangladesh. Infect. Genet. Evol. 2020, 77, 104055. [CrossRef] [PubMed]

13. Prakash, S.; Shukla, S.; Ramakrishna, V.; Mishra, H.; Bhagat, A.K.; Jain, A. Human Parvovirus 4: A harmless bystander or a pathogen of severe acute respiratory illness. Int. J. Infect. Dis. 2020, 90, 21-25. [CrossRef]

14. Manning, A.; Willey, S.J.; Bell, J.E.; Simmonds, P. Comparison of tissue distribution, persistence, and molecular epidemiology of parvovirus B19 and novel human parvoviruses PARV4 and human bocavirus. J. Infect. Dis. 2007, 195, 1345-1352. [CrossRef] [PubMed]

15. Matthews, P.C.; Sharp, C.; Simmonds, P.; Klenerman, P. Human parvovirus 4 'PARV4' remains elusive despite a decade of study. F1000Research 2017, 6, 82. [CrossRef]

16. Simmons, R.; Sharp, C.; McClure, C.P.; Rohrbach, J.; Kovari, H.; Frangou, E.; Simmonds, P.; Irving, W.; Rauch, A.; Bowness, P.; et al. Parvovirus 4 infection and clinical outcome in high-risk populations. J. Infect. Dis. 2012, 205, 1816-1820. [CrossRef]

17. Drexler, J.F.; Reber, U.; Muth, D.; Herzog, P.; Annan, A.; Ebach, F.; Sarpong, N.; Acquah, S.; Adlkofer, J.; Adu-Sarkodie, Y.; et al. Human parvovirus 4 in nasal and fecal specimens from children, Ghana. Emerg. Infect. Dis. 2012, 18, 1650-1653. [CrossRef]

18. Vaisanen, E.; Kuisma, I.; Phan, T.G.; Delwart, E.; Lappalainen, M.; Tarkka, E.; Hedman, K.; Soderlund-Venermo, M. Bufavirus in feces of patients with gastroenteritis, Finland. Emerg. Infect. Dis. 2014, 20, 1077-1079. [CrossRef]

19. Smits, S.L.; Schapendonk, C.M.; van Beek, J.; Vennema, H.; Schurch, A.C.; Schipper, D.; Bodewes, R.; Haagmans, B.L.; Osterhaus, A.D.; Koopmans, M.P. New viruses in idiopathic human diarrhea cases, the Netherlands. Emerg. Infect. Dis. 2014, 20, 1218-1222. [CrossRef]

20. Huang, D.D.; Wang, W.; Lu, Q.B.; Zhao, J.; Guo, C.T.; Wang, H.Y.; Zhang, X.A.; Tong, Y.G.; Liu, W.; Cao, W.C. Identification of Bufavirus-1 and Bufavirus-3 in Feces of Patients with Acute Diarrhea, China. Sci. Rep. 2015, 5, 13272. [CrossRef]

21. CNRvge. Rapport Annuel D'activité 2019, Année D'exercice 2018. Dijon, France. 2019; pp. 31-32. Available online: http: //www.cnr-ve.org/wp-content/uploads/documents/RAPPORT\%20ACTIVITES\%202019.pdf (accessed on 4 September 2021).

22. Pratte-Santos, R.; Miagostovich, M.P.; Fumian, T.M.; Maciel, E.L.; Martins, S.A.; Cassini, S.T.; Keller, R. High prevalence of enteric viruses associated with acute gastroenteritis in pediatric patients in a low-income area in Vitoria, Southeastern Brazil. J. Med. Virol. 2019, 91, 744-750. [CrossRef] [PubMed]

23. Chan, M.C.; Sung, J.J.; Lam, R.K.; Chan, P.K.; Lee, N.L.; Lai, R.W.; Leung, W.K. Fecal viral load and norovirus-associated gastroenteritis. Emerg. Infect. Dis. 2006, 12, 1278-1280. [CrossRef]

24. Mollerup, S.; Fridholm, H.; Vinner, L.; Kjartansdottir, K.R.; Friis-Nielsen, J.; Asplund, M.; Herrera, J.A.; Steiniche, T.; Mourier, T.; Brunak, S.; et al. Cutavirus in Cutaneous Malignant Melanoma. Emerg. Infect. Dis. 2017, 23, 363-365. [CrossRef] [PubMed]

25. Phan, T.G.; Dreno, B.; da Costa, A.C.; Li, L.; Orlandi, P.; Deng, X.; Kapusinszky, B.; Siqueira, J.; Knol, A.C.; Halary, F.; et al. A new protoparvovirus in human fecal samples and cutaneous T cell lymphomas (mycosis fungoides). Virology 2016, 496, 299-305. [CrossRef] [PubMed]

26. Phan, T.; Nagaro, K. Cutavirus: A newly discovered parvovirus on the rise. Infect. Genet. Evol. 2020, 80, 104175. [CrossRef]

27. Yahiro, T.; Wangchuk, S.; Tshering, K.; Bandhari, P.; Zangmo, S.; Dorji, T.; Tshering, K.; Matsumoto, T.; Nishizono, A.; SoderlundVenermo, M.; et al. Novel human bufavirus genotype 3 in children with severe diarrhea, Bhutan. Emerg. Infect. Dis. 2014, 20, 1037-1039. [CrossRef]

28. Ayouni, S.; Estienney, M.; Hammami, S.; Neji Guediche, M.; Pothier, P.; Aouni, M.; Belliot, G.; de Rougemont, A. Cosavirus, Salivirus and Bufavirus in Diarrheal Tunisian Infants. PLoS ONE 2016, 11, e0162255. [CrossRef]

29. Zanella, M.C.; Cordey, S.; Laubscher, F.; Docquier, M.; Vieille, G.; Van Delden, C.; Braunersreuther, V.; Ta, M.K.; Lobrinus, J.A.; Masouridi-Levrat, S.; et al. Unmasking viral sequences by metagenomic next-generation sequencing in adult human blood samples during steroid-refractory/dependent graft-versus-host disease. Microbiome 2021, 9, 28. [CrossRef]

30. Auffret, N.; de Rougemont, A. Détermination des Séroprévalences de Bufavirus et Tusavirus, Deux Nouveaux Protoparvovirus, en Bourgogne; Mémoire du Diplôme D'études Spécialisées de Biologie Médicale. 2020. Available online: https: / / nuxeo.u-bourgogne. fr/nuxeo/nxfile/default/f8bcd3ad-a451-4fd1-9736-907a7b832b0f/file:content/AUFFRET_THESEPHIE_2020-2.pdf (accessed on 4 September 2021).

31. Fryer, J.F.; Delwart, E.; Hecht, F.M.; Bernardin, F.; Jones, M.S.; Shah, N.; Baylis, S.A. Frequent detection of the parvoviruses, PARV4 and PARV5, in plasma from blood donors and symptomatic individuals. Transfusion 2007, 47, 1054-1061. [CrossRef]

32. Panning, M.; Kobbe, R.; Vollbach, S.; Drexler, J.F.; Adjei, S.; Adjei, O.; Drosten, C.; May, J.; Eis-Hubinger, A.M. Novel human parvovirus 4 genotype 3 in infants, Ghana. Emerg. Infect. Dis. 2010, 16, 1143-1146. [CrossRef]

33. Kageyama, T.; Kojima, S.; Shinohara, M.; Uchida, K.; Fukushi, S.; Hoshino, F.B.; Takeda, N.; Katayama, K. Broadly reactive and highly sensitive assay for Norwalk-like viruses based on real-time quantitative reverse transcription-PCR. J. Clin. Microbiol. 2003, 41, 1548-1557. [CrossRef] [PubMed]

34. Le Guyader, F.S.; Parnaudeau, S.; Schaeffer, J.; Bosch, A.; Loisy, F.; Pommepuy, M.; Atmar, R.L. Detection and quantification of noroviruses in shellfish. Appl. Environ. Microbiol. 2009, 75, 618-624. [CrossRef] [PubMed]

35. Freeman, M.M.; Kerin, T.; Hull, J.; McCaustland, K.; Gentsch, J. Enhancement of detection and quantification of rotavirus in stool using a modified real-time RT-PCR assay. J. Med. Virol. 2008, 80, 1489-1496. [CrossRef] [PubMed] 
36. Logan, C.; O'Leary, J.J.; O'Sullivan, N. Real-time reverse transcription PCR detection of norovirus, sapovirus and astrovirus as causative agents of acute viral gastroenteritis. J. Virol. Methods. 2007, 146, 36-44. [CrossRef]

37. Heim, A.; Ebnet, C.; Harste, G.; Pring-Akerblom, P. Rapid and quantitative detection of human adenovirus DNA by real-time PCR. J. Med. Virol. 2003, 70, 228-239. [CrossRef] 